\title{
Lipiodol as a Fiducial Marker for Image-Guided Radiation Therapy for Bladder Cancer
}

Jessica M. Freilich, Philippe E. Spiess, Matthew C. Biagioli, Daniel C. Fernandez, Ellen J. Shi, Dylan C. Hunt, Shilpa Gupta, Richard B. Wilder

Department of Radiation Oncology (JMF, MCB, DCF, DCH, RBW); Department of Urology (PES); Department of Data Quality and Standards (EJS) and Department of Genitourinary Oncology (SG), Moffitt Cancer Center, Tampa, FL, USA

\section{ABSTRACT}

Purpose: To evaluate Lipiodol as a liquid, radio-opaque fiducial marker for image-guided radiation therapy (IGRT) for bladder cancer.

Materials and Methods: Between 2011 and 2012, 5 clinical T2a-T3b N0 M0 stage II-III bladder cancer patients were treated with maximal transurethral resection of a bladder tumor (TURBT) and image-guided radiation therapy (IGRT) to 64.8 Gy in 36 fractions \pm concurrent weekly cisplatin-based or gemcitabine chemotherapy. Ten to $15 \mathrm{~mL}$ Lipiodol, using $0.5 \mathrm{~mL}$ per injection, was injected into bladder submucosa circumferentially around the entire periphery of the tumor bed immediately following maximal TURBT. The authors looked at inter-observer variability regarding the size and location of the tumor bed $\left(\mathrm{CTV}_{\text {boost }}\right)$ on computed tomography scans with versus without Lipiodol.

Results: Median follow-up was 18 months. Lipiodol was visible on every orthogonal two-dimensional kV portal image throughout the entire, 7-week course of IGRT. There was a trend towards improved inter-observer agreement on the $\mathrm{CTV}_{\text {boost }}$ with Lipiodol (p $=0.06$ ). In 2 of 5 patients, the tumor bed based upon Lipiodol extended outside a planning target volume that would have been treated with a radiation boost based upon a cystoscopy report and an enhanced computed tomography (CT) scan for staging. There was no toxicity attributable to Lipiodol.

Conclusions: Lipiodol constitutes a safe and effective fiducial marker that an urologist can use to demarcate a tumor bed immediately following maximal TURBT. Lipiodol decreases inter-observer variability in the definition of the extent and location of a tumor bed on a treatment planning CT scan for a radiation boost.

\section{ARTICLE INFO}

Key words:

Ethiodized Oil; Radiotherapy;

Urinary Bladder

Int Braz J Urol. 2014; 40: 190-7

Submitted for publication:

July 10, 2013

Accepted after revision:

August 28, 2013

\section{INTRODUCTION}

Maximal transurethral resection of a bladder tumor (TURBT) followed by external beam radiation therapy with concurrent chemotherapy constitutes a bladder-sparing treatment option for muscle-invasive bladder cancer. In terms of the radiation therapy, patients are typically treated to the whole bladder to 39.6-45.0 Gy followed by a boost to the tumor bed to a cumulative dose of 64.8-66.0 Gy using 1.8-2.0-Gy daily. In general, a bladder-sparing approach is performed in patients who are medically inoperable or elect not to undergo surgery. Reports show 47-87\% complete 
response rates with bladder-sparing treatment (1). Three-year to 5-year survival rates with an intact bladder have ranged from 37\% to 66\% (1).

Lipiodol (Lipiodol ${ }^{\circledR}$ Ultra-Fluide, Guerbet LLC, Bloomington, IN) consists of ethyl esters of iodized fatty acids of poppy seed oil. Lipiodol was the first iodinated contrast agent and has been in use since 1926. Possible side effects of Lipiodol include an allergic reaction, transient fever during the first few hours following injection, nausea, vomiting, or diarrhea.

Lipiodol may be injected into bladder submucosa circumferentially around the periphery of a tumor bed. The purpose of Lipiodol is to demarcate the tumor bed on a computed tomography (CT) scan to help with the planning of a radiation boost to part of the bladder.

Several groups have shown a high retention rate of Lipiodol in the bladder wall throughout a 6-week to 7-week course of radiotherapy and the feasibility of using Lipiodol as a radio-opaque fiducial marker at sites throughout the bladder (2-5). Since Lipiodol is a liquid, it moves with bladder expansion and contraction. In contrast, Hulshof et al. (6) reported that half of the solid fiducial markers were lost a median of 11.5 days after implantation. Moreover, Mangar et al. (7) were not able to place gold fiducial seeds in the dome of the bladder.

A radiation oncologist normally creates a clinical target volume for a radiation boost (CTV${ }_{\text {boost }}$ ) based upon information including a cystoscopy report and an enhanced CT scan of the pelvis that was obtained for staging (8). Jenkins et al. (9) have recommended that the $\mathrm{CTV}_{\text {boost }}$ should include the tumor bed plus a $10-\mathrm{mm}$ margin in patients with radiological evidence of extravesical disease. In patients with no evidence of extravesical disease, they have suggested that the $\mathrm{CTV}_{\text {boost }}$ should include the tumor bed plus a 6-mm margin. Based upon these recommendations, the $\mathrm{CTV}_{\text {boost }}$ would encompass microscopic disease extension in 90\% of cases.

According to the Radiation Therapy Oncology Group, one may create a planning target volume for a radiation boost, PTV $_{\text {boost }}$, by adding a 20-mm margin on a tumor bed, assuming that Lipiodol was not used in order to help define the tumor bed. This margin accounts for factors such as daily setup error and variation in the position of the tumor bed based upon bladder and rectal filling $(10,11)$.

\section{MATERIALS AND METHODS}

\section{Patients}

After obtaining institutional review board approval to prospectively study Lipiodol, the authors reviewed the medical records of all 5 bladder cancer patients who had undergone Lipiodol-based image-guided radiation therapy (IGRT) at their center between January 1, 2011 and June 30, 2012. All patients had high grade, clinical T2a-T3b No M0 stage II-III urothelial carcinomas. Patients with thyroid disease or a history of iodine allergy did not receive Lipiodol.

\section{Lipiodol demarcation of the tumor bed}

Lipiodol demarcation of the tumor bed was performed under general anesthesia immediately following maximal TURBT. Patients underwent rigid cystoscopy with a 22 French cystoscope by an experienced urologist. There was no difficulty accessing any site within the bladder, including the dome or trigone, with a rigid cystoscope. A 23-gage Chiba-tip needle with a retractable, flexible sheath (Injekt ${ }^{\circledR}$ Cysto Flexible Injection Needle, Cook Medical, Inc., Bloomington, IN) to protect the cystoscope was inserted through the working canal to the tip of the cystoscope. Lipiodol, $0.5 \mathrm{~mL}$ per injection, was placed into bladder submucosa $2-3 \mathrm{~mm}$ from the resection margin in order to outline the entire periphery of the tumor bed. A cumulative total of $10-15 \mathrm{~mL}$ Lipiodol was used, depending on the size of the tumor bed. Roughly 20-30 separate injections were usually administered to demarcate the tumor bed. Fluoroscopic guidance helped to ensure that demarcation of the tumor bed was complete.

\section{Bladder-sparing treatment}

All 5 patients underwent maximal TURBT followed a median of 3 weeks later by intensity modulated radiation therapy (IMRT)/IGRT. IMRT was delivered in order to minimize radiation doses to the recto-sigmoid colon (1). Patients underwent 
IMRT to the whole bladder (clinical target volume) plus a 20-mm margin, which represented the planning target volume (PTV), to 39.6 Gy in 22 fractions over $4 \frac{1}{2}$ weeks. Patients were instructed to have an empty bladder for IMRT to the whole bladder. Patients then underwent an IMRT boost to part of their bladder to a cumulative total dose of 64.8 Gy in 36 fractions over approximately 7 weeks. The tumor bed that had been defined with the help of Lipiodol plus a 15-mm margin constituted the $\mathrm{PTV}_{\text {boost }}$. Patients were instructed to have a full bladder for the IMRT boost. There was no break between the whole bladder and partial bladder irradiation. Prior to each radiotherapy fraction, anterior and right lateral $\mathrm{kV}$ portal images were obtained to set the patient up for IGRT. Four patients received weekly cisplatin-based or gemcitabine chemotherapy concurrently with the IMRT. One patient with a poor performance status due to multiple co-morbidities including angina pectoris underwent IGRT without chemotherapy.

\section{Statistics}

Three radiation oncologists at our center created a $\mathrm{CTV}_{\text {boost }}$ on a CT scan that had been obtained to plan a radiation boost for 2 paired study groups wherein each patient served as a matched control: 1) a no-Lipiodol group, where the CTVboost was based only on a cystoscopy report and an enhanced CT scan of the pelvis that had been obtained pre-Lipiodol for staging, i.e., Lipiodol on the CT scan was ignored; and 2) a Lipiodol group, where the CTV boost was also based on Lipiodol.

We assessed inter-observer variability in demarcation of the tumor bed by 3 different radiation oncologists based on mean percent volume overlap (PVO) of $\mathrm{CTV}_{\text {boost }}$ A similar approach has been used to examine the impact of fiducial markers on inter-observer variability in terms of demarcation of a lumpectomy bed on radiotherapy treatment planning CT scans in breast cancer patients $(12,13)$. PVOs of the $\mathrm{CTV}_{\text {boost }}$ between Radiation Oncologists 1 and 2, Radiation Oncologists 1 and 3, and Radiation Oncologists 2 and 3 were obtained and then averaged. The mathematic formulation of this is mean $((\mathrm{V} 1 \cap \mathrm{V} 2) /(\mathrm{V} 1 \cup \mathrm{V} 2)$, $((\mathrm{V} 1 \cap \mathrm{V} 3) /(\mathrm{V} 1 \cup \mathrm{V} 3),((\mathrm{V} 2 \cap \mathrm{V} 3) /(\mathrm{V} 2 \cup \mathrm{V} 3))$. A mean value for the PVO was calculated for each patient. A Wilcoxon signed-rank test was used to compare the PVOs between groups.

We calculated means and standard deviations for the left-right (LR), cranial-caudal (CC), and anterior-posterior (AP) patient shifts to account for inter-fraction bladder motion $(2,5)$.

\section{Assessment of toxicity}

The Common Terminology Criteria for Adverse Events (CTCAE) version 4.0 grading scheme was used to evaluate the severity of urinary and gastrointestinal toxicity due bladder-sparing therapy.

\section{RESULTS}

Median follow up was 18 months. Median age at diagnosis was 56 years (range, 39 - 65 years). There was one biopsy-proven local relapse in the tumor bed. To date, one patient has died of metastatic disease, one patient is alive with metastatic disease, and 3 patients are alive without evidence of disease.

Lipiodol diffused throughout the tumor bed. Lipiodol was visible on every digitally reconstructed radiograph (Figure 1-A) and every kV image (Figure 1-B) throughout the entire, 7-week course of IGRT. There were no adverse events associated with Lipiodol injections. In terms of treatment-related toxicity, one patient experienced CTCAE version 4.0 acute grade 2 bladder spasms that responded to antispasmodics (Table-1). There was no acute grade $\geq 3$ toxicity or chronic grade $\geq$ 2 toxicity.

Mean daily patient shifts are presented in Table-2. There was a mean \pm standard deviation absolute shift of $2 \pm 2 \mathrm{~mm}$ in the LR direction, $4 \pm$ $3 \mathrm{~mm}$ in the CC direction, and $2 \pm 2 \mathrm{~mm}$ in the AP direction. There was a maximum shift of $14 \mathrm{~mm}$ in the CC direction in one patient. Shifts greater than $5 \mathrm{~mm}$ were made in 4\% (LR), 27\% (CC), and $8 \%$ (AP) of the total number of daily radiotherapy fractions for all patients.

Figure- 2 shows the $\mathrm{CTV}_{\text {boost }}$ with versus without Lipiodol. Lipiodol made it easier to define the extent and location of the tumor bed on a treatment planning CT scan for the radiation boost. The mean PVO was 53\% with Lipiodol versus 39\% 
Figure 1A - Digitally reconstructed radiograph (anterior view) showing Lipiodol contrast demarcating the tumor bed in the anterior and right lateral walls of the bladder.

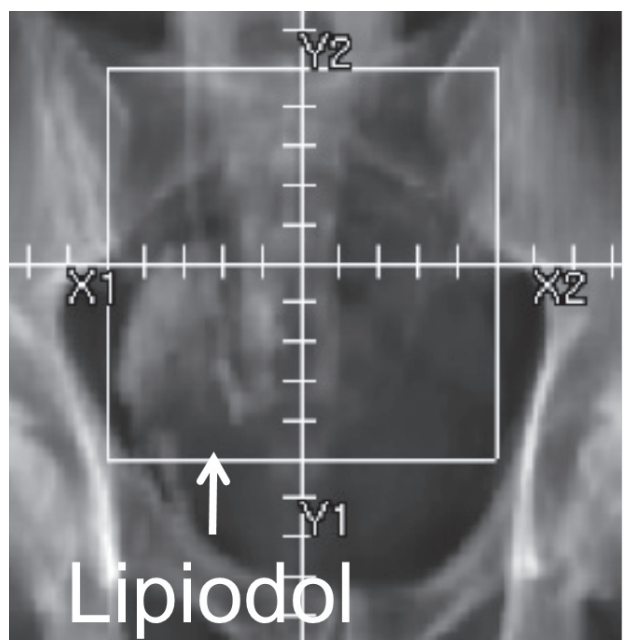

Table 1 - Common Terminology Criteria for Adverse Events version 4.0 acute urinary and gastrointestinal toxicity due to bladder-sparing treatment.

\begin{tabular}{lcc}
\hline Adverse Event & Grade 1 & Grade 2 \\
\hline Fatigue & $100 \%$ & $0 \%$ \\
Cystitis, noninfectious & $100 \%$ & $0 \%$ \\
Diarrhea & $60 \%$ & $0 \%$ \\
Urinary incontinence & $40 \%$ & $0 \%$ \\
Hemorrhoids & $20 \%$ & $0 \%$ \\
Bladder spasms & $0 \%$ & $20 \%$ \\
\hline
\end{tabular}

without Lipiodol (Table-3; $\mathrm{p}=0.06$ ). In two of 5 (40\%) patients, the $\mathrm{CTV}_{\text {boost }}$ based upon Lipiodol extended outside a planning target volume that would have been treated with a radiation boost based upon cystoscopy reports and enhanced CT scans for staging.

\section{DISCUSSION}

The European Association of Urology recommends either an enhanced CT scan or magnetic resonance imaging scan for staging of muscle-invasive bladder cancer (14). Radiation therapy
Figure 1B - kV image (anterior view) showing Lipiodol contrast in the tumor bed in the anterior and right lateral bladder walls.

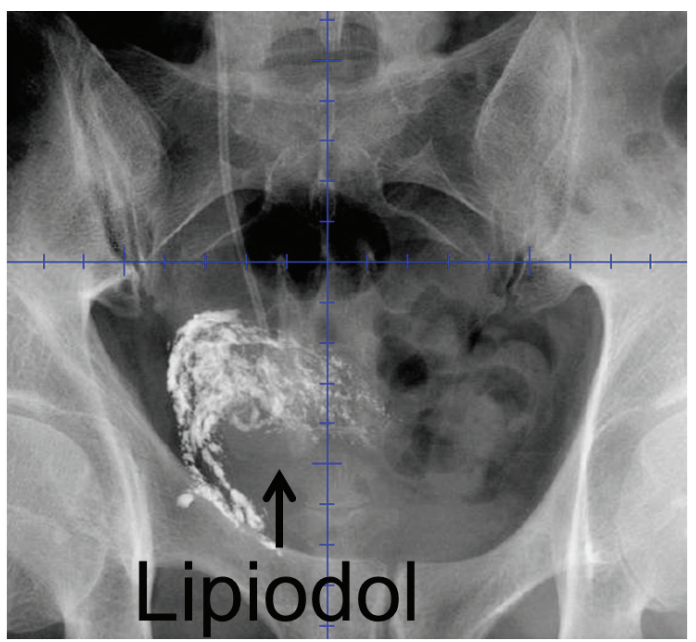

for bladder cancer is commonly planned using an enhanced CT scan of the pelvis.

Bladder cancer is an excellent candidate for IGRT because of substantial motion of the tumor bed during a 6-7 week course of radiotherapy. In this study, bladder motion was most pronounced in the CC dimension (Table-2). Similarly, Meijer et al. (15) found that bladder cancer set up uncertainties varied by direction with the CC dimension having the largest variation. Cranial movement was greater than caudal movement. Also, Fokdal et al. (10) found that bladder movement was most pronounced in the CC direction. Filling volumes of the bladder and rectum had a large impact on bladder movement.

Various studies have examined bladder motion and made recommendations regarding margins on a tumor bed for a $\mathrm{PTV}_{\text {boost }}$. Several groups $(4,15,16)$ have suggested that one should provide a 20-25-mm margin on a tumor bed in order to create a $\mathrm{PTV}_{\text {boost }}$ when no Lipiodol is used to help demarcate the tumor bed. In contrast, Sondergaard et al. (5) have suggested that one only needs a 10-15-mm margin on a tumor bed in order to accurately define a PTV $_{\text {boost }}$ when Lipiodol is used to help define the extent and location of the tumor bed. Furthermore, van Rooijen et al. (17) recommend only a 5-mm margin on a tumor bed in order to create a $\mathrm{PTV}_{\text {boost }}$ when Lipiodol is used. In 
Table 2 - Daily patient shifts (mm) using Lipiodol for IGRT.

\section{A. All patients}

\begin{tabular}{rcccc}
\hline & Mean & Standard deviation & Minimum & Maximum \\
\hline LR shift & 2 & 2 & 0 & 7 \\
CC shift & 4 & 3 & 0 & 14 \\
AP shift & 2 & 2 & 0 & 9 \\
\hline
\end{tabular}

B. Each individual patient

\begin{tabular}{llll}
\hline Mean & Standard deviation & Minimum & Maximum \\
\hline
\end{tabular}

Patient 1:

$\begin{array}{lllll}\text { LR shift } & 3 & 1 & 0 & 5 \\ \text { CC shift } & 2 & 2 & 0 & 7 \\ \text { AP shift } & 3 & 2 & 0 & 9\end{array}$

Patient 2:

$\begin{array}{lc}\text { LR shift } & 1 \\ \text { CC shift } & 10 \\ \text { AP shift } & 3\end{array}$

$\begin{array}{lll}1 & 0 & 4 \\ 2 & 6 & 14 \\ 2 & 1 & 7\end{array}$

Patient 3:

$\begin{array}{ll}\text { LR shift } & 3 \\ \text { CC shift } & 4 \\ \text { AP shift } & 2\end{array}$

2

2

5

7

9

\section{Patient 4:}

$\begin{array}{ll}\text { LR shift } & 2 \\ \text { CC shift } & 4 \\ \text { AP shift } & 2\end{array}$

2

2

2

Patient 5:

$\begin{array}{ll}\text { LR shift } & 2 \\ \text { CC shift } & 2 \\ \text { AP shift } & 1\end{array}$

1

4
14
7

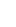

0

0

0

7
8
4 4

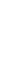


Figure 2 - Axial (A), sagittal (B) and coronal (C) views from a CT scan that was obtained to plan a radiation boost. The CT scan was obtained after Lipiodol had been used to demarcate the tumor bed. The contour with a dark border represents the tumor bed for a radiation boost based upon a cystoscopy report and an enhanced CT scan that had been obtained for staging prior to Lipiodol injection. The contour with a white border represents the tumor bed for a radiation boost based upon Lipiodol contrast as well. Note that Lipiodol contrast extends outside of a tumor bed based upon a cystoscopy report and an enhanced CT scan that had been obtained for staging.

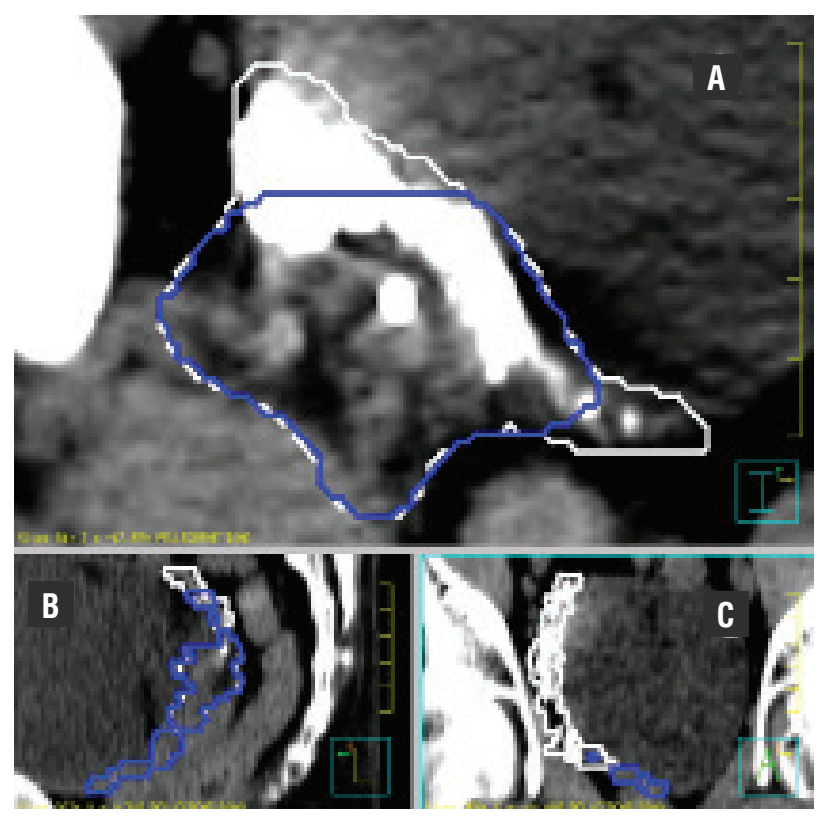

bony structures in many radiation oncology departments. However, IGRT based upon bony structures does not take the substantial daily inter-fraction motion of the bladder into account.

In contrast to the approach taken in Denmark where 3-5 Lipiodol spots with a mean volume of $0.7 \mathrm{cc}$ were typically used to define the periphery of the tumor bed (2-5), Lipiodol was used to demarcate the entire tumor bed in this study. In this report, Lipiodol was visible on every digitally reconstructed radiograph (Figure-1A) and every $\mathrm{kV}$ image (Figure-1B) throughout the entire, 7-week course of IGRT. Similarly, in the study by Chai et al. (2), 34/37 (92\%) Lipiodol deposits that were seen on digitally reconstructed radiographs remained visible on images throughout IGRT. Pos et al. (4) observed that there was a gradual loss of volume of the Lipiodol spots ("washout") over time; however, all of the Lipiodol spots that were present on digitally reconstructed radiographs remained visible throughout radiation therapy. Similarly, Sondergaard et al. (5) observed a relative loss of 24\% in Lipiodol volumes during a 6-week course of radiotherapy. Although there was Lipiodol washout, all Lipiodol spots remained visible throughout radiotherapy.

Reliable tumor bed demarcation on a treatment planning CT scan without Lipiodol is challenging. In many cases, a cystoscopy report and CT scan for staging do not allow a radiation on-

Table 3 - Mean percent volume overlap of CTV $_{\text {boost }}$ with versus without Lipiodol.

\begin{tabular}{|c|c|c|c|}
\hline Patient \# & $\begin{array}{l}\text { Mean Percent Volume Overlap of } \\
\text { CTV }_{\text {boost }} \text { with Lipiodol }\end{array}$ & $\begin{array}{l}\text { Mean Percent Volume Overlap of CTV } \\
\text { without Lipiodol }\end{array}$ & $\begin{array}{l}\text { P-Value for Patients } \\
1-5\end{array}$ \\
\hline 1 & $58 \%$ & $35 \%$ & 0.06 \\
\hline 2 & $72 \%$ & $55 \%$ & \\
\hline 3 & $56 \%$ & $46 \%$ & \\
\hline 4 & $28 \%$ & $14 \%$ & \\
\hline 5 & $51 \%$ & $45 \%$ & \\
\hline Mean: & $53 \%$ & $39 \%$ & \\
\hline
\end{tabular}


cologist to later accurately contour the tumor bed on a radiotherapy treatment planning CT scan. A preferred approach is for an urologist to visualize the tumor bed at the time of cystoscopy and to demarcate the tumor bed with Lipiodol immediately following maximal TURBT. In this report, Lipiodol helped to reduce inter-observer variability regarding the extent and location of the tumor bed on a treatment planning CT scan for a radiation boost (Table-3). In $40 \%$ of patients in this study, the $\mathrm{CTV}_{\text {boost }}$ based upon Lipiodol extended outside of a PTV boost that would have been treated based upon cystoscopy reports and enhanced CT scans without Lipiodol. Similarly, Pos et al. (4) reported that quite often the extent, or sometimes even the location, of Lipiodol spots was different from what the radiation oncologists would have contoured based upon cystoscopy reports and CT scans without Lipiodol.

In accordance with reports by others $(4,15)$, Lipiodol injections were well-tolerated in this study. There were no adverse events due to Lipiodol injections.

The main strength of this study is that it is the first one to look at inter-observer variability regarding the extent and location of the tumor bed on CT scans with versus without Lipiodol (Table-3). The main weakness of this study is its small sample size. This was due to the infrequent utilization of bladder-sparing therapy at our referral center due to patient treatment preference. Another weakness is that intra-fraction movement of the bladder was not assessed in this study.

\section{CONCLUSIONS}

Bladder motion between daily radiotherapy treatments occurs primarily in the CC dimension and is substantial. Lipiodol constitutes a safe and effective fiducial marker that helps one to define the extent and location of a tumor bed for a radiation boost. Consequently, Lipiodol may lead to better local control and progression-free survival through more accurate targeting of the tumor bed with radiotherapy. We recommend that future prospective trials involving radiotherapy for muscle-invasive bladder cancer should include Lipiodol as a fiducial marker to not only corroborate its safety but also to assess its potential to improve local control and progression-free survival.

\section{ABBREVIATIONS}

$\mathrm{AP}=$ Anterior-posterior

$\mathrm{CC}=$ Cranial-caudal

$\mathrm{CTV}_{\text {boost }}=$ Clinical target volume for a radiation boost

CTCAE $=$ Common Terminology Criteria for Adverse Events

$\mathrm{CT}=$ Computed tomography

Gy $=$ Gray

IGRT = Image-guided radiation therapy

IMRT = Intensity modulated radiation therapy

LR = left-right

TURBT = Maximal transurethral resection of a bladder tumor

PVO = Percent volume overlap

$\mathrm{PTV}_{\text {boost }}$ planning target volume for a radiation boost

\section{CONFLICT OF INTEREST}

None declared.

\section{REFERENCES}

1. Biagioli MC, Fernandez DC, Spiess PE, Wilder RB: Primary bladder preservation treatment for urothelial bladder cancer. Cancer Control. 2013; 20: 188-99.

2. Chai $X$, van Herk M, van de Kamer JB, Remeijer P, Bex A, Betgen $A$, et al:: Behavior of lipiodol markers during image guided radiotherapy of bladder cancer. Int J Radiat Oncol Biol Phys. 2010; 77: 309-14.

3. Meijer GJ, van der Toorn PP, Bal M, Schuring D, Weterings $\mathrm{J}$, de Wildt M: High precision bladder cancer irradiation by integrating a library planning procedure of 6 prospectively generated SIB IMRT plans with image guidance using lipiodol markers. Radiother Oncol. 2012; 105: 174-9.

4. Pos F, Bex A, Dees-Ribbers HM, Betgen A, van Herk M, Remeijer P: Lipiodol injection for target volume delineation and image guidance during radiotherapy for bladder cancer. Radiother Oncol. 2009; 93: 364-7.

5. Søndergaard J, Olsen Kø, Muren LP, Elstrøm UV, Grau C, Høyer M: A study of image-guided radiotherapy of bladder cancer based on lipiodol injection in the bladder wall. Acta Oncol. 2010; 49: 1109-15. 
6. Hulshof MC, van Andel G, Bel A, Gangel P, van de Kamer JB: Intravesical markers for delineation of target volume during external focal irradiation of bladder carcinomas. Radiother Oncol. 2007; 84: 49-51.

7. Ferrer M, Suárez JF, Guedea F, Fernández P, Macías V, Mariño A, et al.: Health-related quality of life 2 years after treatment with radical prostatectomy, prostate brachytherapy, or external beam radiotherapy in patients with clinically localized prostate cancer. Int J Radiat Oncol Biol Phys. 2008; 72: 421-32.

8. Lang EK, Nguyen Q, Zhang K: Enhanced computed tomograms provide accurate assessment of local extension and stage of bladder carcinoma. Int Braz J Urol. 2012; 38: 129-31.

9. Jenkins $P$, Anjarwalla $S$, Gilbert $H$, Kinder R: Defining the clinical target volume for bladder cancer radiotherapy treatment planning. Int J Radiat Oncol Biol Phys. 2009; 75: 1379-84.

10. Fokdal L, Honoré $H$, Høyer M, Meldgaard P, Fode K, von der Maase $\mathrm{H}$ : Impact of changes in bladder and rectal filling volume on organ motion and dose distribution of the bladder in radiotherapy for urinary bladder cancer. Int J Radiat Oncol Biol Phys. 2004; 59: 436-44.

11. Pos FJ, Koedooder K, Hulshof MC, van Tienhoven G, González González D: Influence of bladder and rectal volume on spatial variability of a bladder tumor during radical radiotherapy. Int J Radiat Oncol Biol Phys. 2003; 55: 835-41.

12. Landis DM, Luo W, Song J, Bellon JR, Punglia RS, Wong JS, et al.: Variability among breast radiation oncologists in delineation of the postsurgical lumpectomy cavity. Int J Radiat Oncol Biol Phys. 2007; 67: 1299-308.
13. Shaikh T, Chen T, Khan A, Yue NJ, Kearney T, Cohler A, et al.: Improvement in interobserver accuracy in delineation of the lumpectomy cavity using fiducial markers. Int J Radiat Oncol Biol Phys. 2010; 78: 1127-34.

14. Stenzl A, Cowan NC, De Santis M, Kuczyk MA, Merseburger AS, Ribal MJ, et al.: Treatment of muscle-invasive and metastatic bladder cancer: update of the EAU guidelines. Eur Urol. 2011; 59: 1009-18.

15. Meijer GJ, Rasch C, Remeijer P, Lebesque JV: Threedimensional analysis of delineation errors, setup errors, and organ motion during radiotherapy of bladder cancer. Int $\mathrm{J}$ Radiat Oncol Biol Phys. 2003; 55: 1277-87.

16. Turner SL, Swindell R, Bowl N, Marrs J, Brookes B, Read $G$, et al.: Bladder movement during radiation therapy for bladder cancer: implications for treatment planning. Int $\mathrm{J}$ Radiat Oncol Biol Phys. 1997; 39: 355-60.

17. van Rooijen DC, Pool R, van de Kamer JB, Hulshof MC, Koning CC, Bel A: Independent position correction on tumor and lymph nodes; consequences for bladder cancer irradiation with two combined IMRT plans. Radiat Oncol. 2010; 5:53.
Correspondence address: Richard B. Wilder, MD Department of Radiation Oncology Moffitt Cancer Center 12902 Magnolia Drive Tampa, FL 33612, U.S.A. Fax: +1 813 745-7231

E-mail: richard.wilder@moffitt.org 